\title{
Efficacy of rehabilitation program in addition to pharmacological treatment during 8 months in Parkinson patients
}

\author{
Jesús Seco Calvo ${ }^{1}$, Inés Gago Fernández ${ }^{\star *}$, Juan Azael Herrero Alonso ${ }^{3}$, Javier González \\ Gallego $^{1}$, Nuria Garatachea Vallejo ${ }^{1}$ \\ ${ }^{1}$ Institute of Biomedicine (IBIOMED), University of León, León, Spain; \\ ${ }^{2}$ Faculty of Health Sciences, University of León, Ponferrada, Spain; ${ }^{*}$ Corresponding Author: ines.gago@unileon.es \\ ${ }^{3}$ Research Center on Physical Disability, ASPAYM Castilla y León, Valladolid, Spain
}

Received 18 September 2012; revised 9 October 2012; accepted 15 November 2012

\begin{abstract}
Objective: To compare 8-month effects of medical treatment plus rehabilitation on UPDRS scores of parkinsonian patients with that of medical treatment without rehabilitation. Design: Longtudinal randomized study. Participants: 27 parkinsonian patients (69.50 \pm 10.34 years). We divided our patient into two groups: control group ( $n=9$, received only medication therapy) and experimenttal group ( $n=18$, received physiccal therapy and medication therapy). Intervention: The 8-month exercise interventions were twice-weekly $90-\mathrm{min}$ sessions in group. UPDRS scales were measured before and after the interventions. Results: Twofactor ANOVA analyses revealed a significant main effect from rehabilitation $(p<0.01)$ on UPDRS motor, ADL, and total, but not on UPDRS mental $(p>0.05)$. Post-hoc analysis shows that UPDRS motor increased in control group (+37\%) meanwhile decreased in experimental group $(-17 \%)$. UPDRS ADL increased significantly more in control group (+26\%) than experimental group $(+5 \%)$. UPDRS total increased in control group (+33\%) meanwhile decreased in experimental group $(-11 \%)$. Conclusions: The results of the study suggest that exercise interventions should be a necessary ongoing adjunct to parkinson's disease medication.
\end{abstract}

Keywords: Parkinson; Rehabilitation; UPDRS

\section{INTRODUCTION}

Parkinson's disease (PD) is a progressive neurological disease which, despite an optimum medical treatment, results in a progressive loss of the patient's functional abilities and in a decrease in his/her capability to lead an independent life. The clinical hallmarks or neurologic symptoms of the disease include difficulty in initiating movement (akinesia); slowness and difficulty in maintaining movement (bradykinesia); reduced ability to switch between different coordination patterns (set shifting); stiffness in arms, legs and trunk (rigidity); postural instability and a pathologic tremor at approximately $5-6 \mathrm{~Hz}$ [1]. PD is characterized by insidious onset. The pharmacological treatment of PD mainly comprises dopamine replacement therapy (levodopa plus carbidopa [Sinemet], levodopa plus benserazide [Madopar]) and/or dopamine agonists (pergolide [Permax] and/or bromocriptine [Parlodel]), whereas surgery is sometimes performed. Motor disturbances related to PD can lead to a reduction in functional independence. Functional independence is related to the capacity to perform activities of daily living (ADL) independently.

Physical Therapy may serve as an important adjunct to the available pharmacological and neurosurgical treatment regimes, in view of the fact that most pharmacological and surgical treatments are able to reduce, but not eliminate the neurological deficits of bradykinesia, rigidity and freezing. In addition, pharmacological treatment is often insufficient to improve non-dopaminergic symptoms such as lack of balance control and resulting falls. Therefore, regular physical exercise therapy sessions, supported by a physical therapist, are warranted for most patients with PD [1]. A review of the literature have concluded that, through exercise, patients with PD improve their physical performance and the execution of ADL [2].

Physical activity programs for PD patients that focus on improvements in functional capacity and mobility vary according to the type of proposed activity, whether it will be practiced by individuals or in a group, the program's duration, the duration and frequency of weekly sessions, and type of evaluation. Such programs include intensive sports training [3], treadmill training with body 
weight support [4], resistance training [5-6], aerobic exercise [7], alternative forms of exercise [8], home-based exercise intervention [9], and the practice of movement strategies [10].

Within this context, the purpose of this study was to verify the efficacy of a 8-month physiotherapy program for patients with idiopathic $\mathrm{PD}$, in addition to anti-parkinsonian pharmacologic treatment on ADL, and motor and mental activity. Two interventional programs, a pharmacologic program and a pharmacologic plus physiotherapy program, were applied to PD patients. The effecttiveness of the programs was judged relative to the diseases' severity of the patient, which were measured by UPDRS (section I-Mentation (Mental Activity), Behavior, and Mood; section II-Activities of Daily Living (ADL); and section III-Motor Examination) which is the most accepted tool used in clinical research for measuring the longitudinal course of PD.

\section{MATERIALS and METHODS}

\subsection{Subjects}

Patients with idiopathic Parkinson's disease, recruited through personal letters from Astorga Parkinson Disease Patients Association (Astorga, Spain), volunteered participate in the study. An extensive medical screening was performed by a physician who checked the inclusion and exclusion criteria (Table 1). Of the 32 patients initially included, 27 (13 females and 14 males) completed the baseline and treatments period. After patients were carefully informed about the design of the study, they signed a written informed consent before participation. The research was conducted according to the declaration of Helsinki and was approved by the Ethics Committee of the University of León (Spain). Mean \pm SD disease duration was $11.4 \pm 1.6$ years. All subjects were taken: L-dopa, dopamine-agonist and amantadine. All pharmacological treatments were kept at a stable dosage for 30 days prior to study entry and throughout the study. All subjects were required to take their medications at the same time of day for all assessment sessions.

\subsection{Experimental Design}

Subjects were randomly allocate to either (A) medical treatment plus "best practice" physiotherapy (experimenttal group: $\mathrm{N}=18$; aged $69.5 \pm 10.3$ years) or to (B) medical treatment without physiotherapy (control group: $\mathrm{N}=9$; aged $67.8 \pm 4.9$ years). Baseline data (pre-test) were collected during two testing sessions. During one testing session a neurologist attempted to assess when the patients were in "on" phase of their PD (i.e., when moto symptoms were reduced) and during another testing session when the patients were in "off" phase. Similar testing sessions were repeated 8 months after (post-test) the
Table 1. Inclusion and exclusion criteria.
Inclusion criteria

- Idiopathic Parkinson's disease according to the UK PDS Brain Bank criteria [11]

- Stable reaction to anti-Parkinson medication.

- Hoehn and Yahr stage I, II or • III.

- At least one mobility-related activity limitation within the core areas of physiotherapy practice in PD (gait, balance and posture).
Exclusion criteria

Severe cognitive impairment, defined by Mini-Mental State Examination score $\leq 24$ [12], or presence of psychiatric impairments

Severe neurologic, cardiopulmonary, or orthopaedic disorders.

Have participated in a physical activity or rehabilitation program in the previous 4 month experimental/control period. (See Figure 1: study design and flow of participants through each stage of the trial).

\subsection{Physiotherapy Program}

The physiotherapy program included different sequences of exercises and was specifically designed to address three objectives: to improve motor skills, to correct abnormal postures and to increase motor dexterity. The program was administered by experienced physical therapists mainly in group (twice per week), except monthly individual session. The group sessions lasted 90 minutes. Each session included cardiovascular warm-up (5 min), stretching exercises $(15 \mathrm{~min})$, strengthening exercises in a functional context $(15 \mathrm{~min})$, functional training (15 min), gait training over ground and on a treadmill with external auditory cueing (15 min), balance training and recreational games $(15 \mathrm{~min})$, and relax exercise $(10 \mathrm{~min})$. All patients received a monthly individual session of relaxation massage: surface rubbing, kneading, etc. during 45 minutes.

\subsection{Unified PD Rating Scale (UPDRS)}

The UPDRS was originally developed to serve as an assessment of the severity of the disease [13]. Nowadays the UPDRS is the most accepted tool used in clinical research for measuring the longitudinal course of PD $[14,15]$. The UPDRS consists of 6 sections. Only the sections I to III were used for this study: section I (UPDRS mental)-Mentation (Mental Activity), Behavior, and Mood; section II (UPDRS ADL) - Activities of Daily Living (ADL); and section III (UPDRS motor)Motor Examination. Mentioned sections are scored on a 5-points Likert scale from 0 to 4 , with 0 representing "no impairment" and 4 representing "marked Impairment". The UPDRS total score were also calculated and reflects performance on these 3 sections (total possible score = 124), with lower scores showing less disability. Sections of the UPDRS are scored and reported separately. All patients were evaluated by a neurologist with expertise in movement's disorders. 


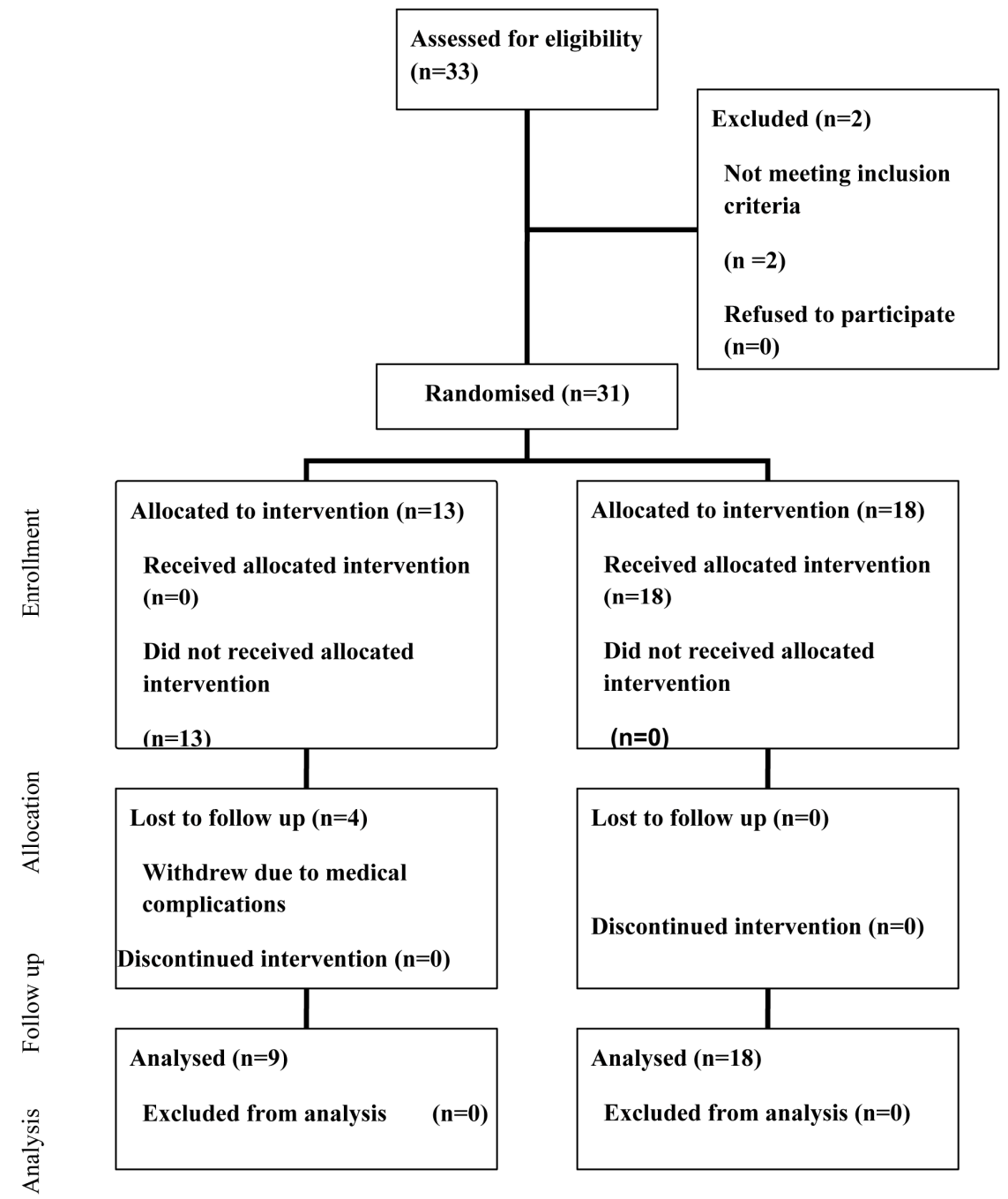

Figure 1. CONSORT Flow Diagram. Study design and flow of participants through each stage of the trial.

\subsection{Statistical Analysis}

The data are presented as means \pm standard deviation (SD). All measures were normally distributed, as determined by the Shapiro-Wilks test. Percentage of variation was calculated for each variable as: [(posttest-pretest) $* 100$ /pretest]. Statistical analysis was performed using an analysis of variance (ANOVA): 2 (group) $\times 2$ (phase). A Bonferroni post hoc test was used in all pairwise comparisons when a significant result was found. The significance level was set at $\mathrm{P}<0.05$ for all the comparisons.

\section{RESULTS AND DISCUSSION}

\subsection{Results}

Percentage of variation respect to basal value of each variable are presented in Table 2 The results of the two-factor ANOVA for revealed a significant group main effect for UPDRS motor $\left(\mathrm{p}<0.001 ; \eta^{2}=0.519\right)$, UPDRS ADL $\left(\mathrm{p}<0.01 ; \eta^{2}=0.151\right)$ and UPDRS total $(\mathrm{p}$
$<0.001 ; \eta^{2}=0.516$ ), but no for UPDRS mental ( $\mathrm{p}>$ $0.05)$. Post-hoc analysis shows that UPDRS motor in-

Table 2. Percentage of variation of each variable respect basal value. C: control, E: experimental.

\begin{tabular}{lcccccc}
\hline & & & \multicolumn{2}{c}{ Phase ON } & \multicolumn{2}{c}{ Phase OFF } \\
\cline { 3 - 6 } & & Croup & Mean & SD & Mean & SD \\
\hline UPDRS mental & E & 7.8 & 16.5 & 2.0 & 9.5 \\
& C & 46.8 & 6.9 & 28.2 & 4.7 \\
UPDRS motor & E & -20.1 & 37.6 & -15.8 & 23.7 \\
& C & 39.4 & 11.6 & 13.1 & 9.8 \\
UPDRS ADL & E & 8.2 & 31.0 & 2.5 & 25.8 \\
& C & 42.6 & 4.7 & 23.8 & 3.9 \\
UPDRS total & E & -12.4 & 30.1 & -11.2 & 20.5 \\
\hline
\end{tabular}


creased in control group $(+37 \%)$ meanwhile decreased in experimental group $(-17 \%)$. UPDRS ADL increased significantly more in control group $(+26 \%)$ than experimental group $(+5 \%)$. UPDRS total increased in control group $(+33 \%)$ meanwhile decreased in experimental group $(-11 \%)$.

The results of the two-factor ANOVA for UPDRS ADL revealed a significant main phase effect $(\mathrm{p}<0.05$; $\left.\eta^{2}=0.094\right)$. Bonferroni post-hoc comparison indicated that UPDRS ADL increased more in ON $(+24 \%)$ than OFF $(+8 \%)$ phase.

There were no significant group $\times$ phase interaction effect $(p>0.05)$.

\subsection{Discussion}

The main findings of the present study was that pharmacological treatment plus "best practice" physiotherapy program induced significant improvements on UPDRS motor and total meanwhile pharmacological treatment without physiotherapy was insufficient to induce any positive change in these UPDRS scales. In addition, although UPDRS ADL increased in both groups, pharmacological plus physiotherapy treatment induced greater increments than pharmacological treatment only.

It is well documented that physical activity should be a component of healthy everyday life for everyone [16], and there is a consensus amongst researchers about the short-term benefits of exercise interventions for people with PD [9, 10, 13-17]. A number of intervention studies have been carried out to investigate the efficacy of PT for patients, in addition to anti-parkinsonian pharmacologic treatment, but there is also a need for longer term studies (over 1 year) [2]. At the end of the present study, the experimental group experienced a decrement of $17 \%$ in UPDRS motor, and $11 \%$ in UPDRS total, meanwhile UPDRS ADL only increase a 5\%. Therefore, the results of our study indicate that the improvements obtained during the intervention stage ( 8 months) are retained long term. In order to prolong the benefits provided by these interventions, people with PD should practice exercises on an everyday basis. PD patients enrolled in exercise interventions with durations longer than six months, regardless of exercise intensity, have shown significant gains in functional balance and mobility as compared to programs of only two-week [17] or ten-week [9] durations. Inactivity has been responsible for the increment in ADL performance lost while exercise can stimulate dopamine synthesis in remaining dopaminergic cells.

These results of the study support the use of physical therapy as adjuncts to pharmacological treatment for people with PD. Inactivity by PD is responsible for incremental losses in ADL performance, while exercise can stimulate dopamine synthesis in remaining dopa- minergic cells [18]. The association between the disease's progression, undesired effects of anti-Parkin sonian medication, and inactivity can reduce patients' quality of life in a cyclical, reactive manner, which some authors refer to as accelerated aging [19]. PD interferes with various aspects of quality of life, particularly those related to physical and social functioning; in addition, we think that that the improvements obtained during the inte- rvention stage in both aspects constitute a benefit of quality of life for people with PD.

Since no guidelines have yet disclosed what is the recommended content (dosing, techniques) and timing of exercise interventions (when to start, how long to continue) [20], the performed program had an important comprehensive character. Cardiovascular warm-up, stretching exercises, strengthening exercises in a functional context, functional training, gait training over ground, balance training and recreational games, and relax exercise were included in the physiotherapy program. Kwakkel et al. [1] affirm that future studies should involve a stable PD medication regime as well as standardized assessment times (i.e., assessment always at the same time after medication intake, or standardized for on and off periods), and our study.

The optimum form that such exercise practices should take is not yet clear, and a variety of activities has been suggested. For example, Falvo et al. [21] recommended resistive exercises, while Hackney and Earhart [8] recommended Tango dance. However, the patient's interest and pleasure should be considered, as well as the inclusion of outpatient settings for people with PD [8,9]. Apart from traditional treatments, a series of supplementary methods are also applied, such as Qigong. Studies in such line by Schmitz-Hübsch et al. [11] demonstratedafter 3, 6 and 12 months - that there were more patients whose symptoms improved in the Qigong group than in control group within a 3 and 6-month period $(\mathrm{P}=0.0080$ for 3 months and $\mathrm{P}=0.0503$ for 6 months; using the Fisher's exact test); depression scores diminished in both groups, while the incidence of non-motor symptoms only diminished in the treatment group.

\section{CONCLUSIONS}

People with PD can benefit from physical therapy, since they can help facilitate and prolong the performance of ADL, and, consequently, quality of life. Definitively, since Jöbges et al. [12] demonstrated the clinical relevance of rehabilitation programs for patients of $\mathrm{PD}$ is estimated to be sufficient if the following seven criteria are met: effectiveness, everyday life relevance, long-term effect, therapy frequency+setting, duration of therapy units, quality of live, timing of assessment + medication; In conclusion, the results of the study suggest that exer- 
cise interventions should be a necessary ongoing adjunct to PD medication. That is, exercise practice should be promoted not only as a therapy, but also as an activity of a healthy patient lifestyle.

\section{CONFLICT OF INTERESTS}

All the authors contributed substantially to the conception and design of this study, and to the revision of this article.

Approved by the Bioethics Committee, University of León, Spain.

No benefits in any form have been or will be received from a commercial party related directly or indirectly to the subject of this article.

The authors do not have conflicts of interest to report. No sources of funding were used to assist in the preparation of this manuscript. The authors have no conflicts of interest that are directly relevant to the content of this article.

\section{ACKNOWLEDGEMENTS}

There was no funding source. The authors would like to thank the members of the Association of PD Patients from Astorga and its Region (Spain) for their interest and collaboration, and of the physicians and physical therapists who participated in the study.

\section{REFERENCES}

[1] Kwakkel, G., de Goede, C.J. and van Wegen, E.E. (2007) Impact of physical therapy for Parkinson's disease: A critical review of the literature. Parkinsonism \& Related Disorders, 13, S478-S487. doi:10.1016/S1353-8020(08)70053-1

[2] Crizzle, A.M. and Newhouse, I.J. (2006) Is physical exercise beneficial for persons with Parkinson's disease? Clinical Journal of Sport Medicine, 16, 422-425. doi:10.1097/01.jsm.0000244612.55550.7d

[3] Reuter, I., Engelhardt, M., Stecker, K. and Baas, H. (1999) Therapeutic value of exercise training in Parkinson's disease. Medicine \& Science in Sports \& Exercise, 31, 15441549. doi:10.1097/00005768-199911000-00008

[4] Miyai, I., Fujimoto, Y., Ueda, Y., Yamamoto, H., Nozasaki, S., Saito, T., et al. (2000) Treadmill training with body weight support: Its effect on Parkinson's disease. Archives of Physical Medicine and Rehabilitation, 81, 849852. doi:10.1053/apmr.2000.4439

[5] Dibble, L.E., Hale, T.F., Marcus, R.L., Gerber, J.P. and LaStayo, P.C. (2009) High intensity eccentric resistance training decreases bradykinesia and improves quality of life in persons with Parkinson's disease: A preliminary study. Parkinsonism \& Related Disorders, 15, 752-757. doi:10.1016/j.parkreldis.2009.04.009

[6] Scandalis, T.A., Bosak, A., Berliner, J.C., Helman. L.L. and Wells, M.R. (2001) Resistance training and gait function in patients with Parkinson's disease. American Journal of Physical Medicine \& Rehabilitation, 80, 38-43. doi:10.1097/00002060-200101000-00011

[7] Bergen, J.L., Toole, T., Elliott, R.G., Wallace, B., Robinson, K. and Maitland, C.G. (2002) Aerobic exercise in- tervention improves aerobic capacity and movement initiation in Parkinson's disease patients. NeuroRehabilitation, 17, 161-168.

[8] Hackney, M.E. and Earhart, G.M. (2009) Health-related quality of life and alternative forms of exercise in Parkinson disease. Parkinsonism \& Related Disorders, 15, 644648. doi:10.1016/j.parkreldis.2009.03.003

[9] Nocera, J., Horvat, M. and Ray, C.T. (2009) Effects of home-based exercise on postural control and sensory organization in individuals with Parkinson disease. Parkinsonism \& Related Disorders, 15, 742-745. doi:10.1016/j.parkreldis.2009.07.002

[10] Hirayama, M.S., Gobbi, S., Gobbi, L.T and Stella, F. (2008) Quality of life (QoL) in relation to disease severity in Brazilian Parkinson's patients as measured using the WHOQOL-BREF. Archives of Gerontology and Geriatrics, 46, 147-160. doi:10.1016/j.archger.2007.03.007

[11] Schmitz-Hübsch, T., Pyfer, D., Kielwein, K., Fimmers, R., Klockgether, T and Wüllner, U. (2006) Qigong exercise for the symptoms of Parkinson's disease: A randomized, controlled pilot study. Movement Disorders, 21,543-548. doi: $10.1002 / \mathrm{mds} .20705$

[12] Jöbges, E., Spittler-Schneiders, H., Renner, C. and Hummelsheim, H. (2007) Clinical relevance of rehabilitation programs for patients with idiopathic Parkinson syndrome. II: Symptom-specific therapeutic approaches. Parkinsonism \& Related Disorders, 13, 203-213. doi:10.1016/j.parkreldis.2006.07.018

[13] Fahn, S. and Elton, R.L. (1987) Unified Parkinson's disease rating scale. In: Fahn, S., Marsden, C.D., Calne, D. and Goldstein, M., Eds., Recent Developments in Parkinson's Disease, Macmillan Health Care Information, Florham Park, 153-163.

[14] Richards, M., Marder, K., Cote, L. and Mayeux, R. (1994) Interrater reliability of the unified Parkinson's disease rating scale motor examination. Movement Disorders, 9, 89-91. doi: $10.1002 / \mathrm{mds} .870090114$

[15] Van Hilten, J.J., van der Zwan, A.D., Zwinderman, A.H. and Roos, R.A. (1994) Rating impairment and disability in Parkinson's disease: Evaluation of the unified Parkinson's disease rating scale. Movement Disorders, 9, 84-88. doi: $10.1002 / \mathrm{mds} .870090113$

[16] Taylor, A.H., Cable, N.T., Faulkner, G., Hillsdon, M., Narici, M. and Van Der Bij, A.K. (2004) Physical activity and older adults: A review of health benefits and the effectiveness of interventions. Journal of Sports Sciences, 22, 703-725. doi:10.1080/02640410410001712421

[17] Morris, M.E., Iansek, R. and Kirkwood, B. (2009) A randomized controlled trial of movement strategies compared with exercise for people with Parkinson's disease. Movement Disorders, 24, 64-71. doi:10.1002/mds.22295

[18] Sutoo, D. and Akiyama, K. (2003) Regulation of brain function by exercise. Neurobiology of Disease, 13, 1-14. doi:10.1016/S0969-9961(03)00030-5

[19] Glendinning, D.S. and Enoka, R.M. (1994) Motor unit behavior in Parkinson's disease. Physical Therapy, 74, 61-70. 
[20] Ceravolo, M.G. (2009) Rehabilitation goals and strategies in Parkinson's disease. European Journal of Physical and Rehabilitation Medicine, 45, 205-208.

[21] Falvo, M.J., Schilling, B.K and Earhart, G.M. (2008)
Parkinson's disease and resistive exercise: Rationale, review, and recommendations. Movement Disorders, 23, 1-11. doi: $10.1002 / \mathrm{mds} .21690$ 